\title{
An Optimized Approach to Evaluate Late Stage Degradation of Absorbable Mesh Implants and Associated Tissue Response
}

\author{
Emilie Payet ${ }^{1}$, Antoine Alves ${ }^{2}$, Cécile Beausseron-Valentin ${ }^{1}$, Thierry Brune ${ }^{1}$, Amandine Radlovic ${ }^{1}$ and \\ Yves Bayon ${ }^{1 *}$ \\ ${ }^{1}$ Medtronic - Sofradim Production \\ ${ }^{2}$ NAMSA, France
}

*Corresponding author: Yves Bayon, Medtronic - Sofradim Production, 116 av du Formans, 01600 Trevoux, France

ARTICLE INFO

Received: 幽 December 11, 2019

Published: 慧December 18, 2019

Citation: Emilie Payet, Antoine Alves, Cécile Beausseron-Valentin, Thierry Brune, Amandine Radlovic, Yves Bayon. An Optimized Approach to Evaluate Late Stage Degradation of Absorbable Mesh Implants and Associated Tissue Response. Biomed J Sci \& Tech Res 24(1)-2019. BJSTR. MS.ID.003987.

Keywords: Absorbable Polymers; Polylactide; Mesh Implant; Degradation; Histology; Local Tissue Effects; Foreign Body Reaction

\section{ABSTRACT}

Absorbable implantable medical devices to be approved for clinical use are required to be systematically evaluated for biocompatibility before being considered for clinical use approval, even if they are made of well-known and used polymers. For long term absorbable implants, the foreign body reaction should be assessed during the full implantation time, at each main degradation stage as defined International Standard ISO-10993 Biological evaluation of medical devices-Part 6. The present study was performed on absorbable polyester meshes made of poly(L-lactide) and poly(L-lactide) trimethylene carbonate and intended to be used for soft tissue repair. Studies should be done on native materials and, possibly, on predegraded materials for slow absorbable polymers. When pre-degraded at around 50\% in mass during in vitro pre-degradation, meshes lose their structural integrity and original form. The resulting meshes particles were implanted subcutaneously in rabbits either as free particles (FP) or as particles homogeneously embedded in collagen film discs (PIC).

Local tissue effects and mesh degradation were macroscopically and histologically investigated up to 52 weeks. FP tended to present an heterogenous distribution among the implantation sites, leading to compacted material and resulting in fibrous encapsulation, signs of necrosis and poor cell infiltration. Rapidly after implantation, FP piled to each other with limited body fluid circulation that slowed the polymer degradation. PIC led to a more homogenous distribution fo well interspaced particles. At 26 weeks, particles were well integrated into tissues and delineated by a normal foreign body reaction. Some reduction of the diameter of the particles has been observed. At 52 weeks, only intracytoplasmic material remained visible which was indicative of end-stage absorption of the polymers. The formulation of the pre-degraded mesh in a collagen film prevented particles aggregation and allowed a more appropriate and predictive assessment of local tissue effects and degradation.

In conclusion, the evaluation of foreign body of reaction, elicited by absorbable polymeric materials during their implantation life can be accelerated by using predegraded materials. But they should be appropriately formulated to adequately recapitulate the different degradation stages, especially the latest, occurring naturally with native materials.

\section{Introduction}

Degradable polymers, such as polyesters, have been used in medical devices for decades [1,2]. Polylactic acid (PLA) and PLAbased copolymers have been proved to be biocompatible $[3,4]$.
Although degradable polyesters are most generally safe polymers, their degradation profile and the induced local tissue reaction may vary according to their chemical properties and manufacturing pro- 
cess. For example, thermal treatments such as extrusion can drastically change the crystallinity of the polymers and, then, their degradation profile independently of their chemistry [5,6]. Sterilization impact on degradable polyesters is also well known [4]. Therefore, any new absorbable polyester device needs first to be fully characterized (e.g. chemical composition, molar mass, crystallinity, ...) and checked for stability in packaging. Clinical indications of degradable polyesters are also potentially wide, meaning fate and, particularly, degradation kinetic and associated local tissue effects, after implantation which can be different due to varying

a) Intensity of foreign body reaction.

b) pH in microenvironment and

c) Size and shape of the implant. Then, although degradable polyesters are very well known and described in the literature since the 60's, safety and degradation profile should be systematically evaluated for any clinical development of implants made of degradable polyesters. And this is strongly required by any medical device regulation agencies for market approval, such as FDA and EU notified bodies. For example, the last revision of European guidelines for Medical Device, Meddev 2.7/1 revision 4 (June 2016) gives strict indications for the demonstration of equivalence of medical devices to support same safety profile and clinical efficacy:

a) Same clinical indications.

b) Similar device design and properties (eg. tensile strength, viscosity, surface characteristics, mass, surface texture, porosity, particle size).

c) Similar principles of operation and critical performance requirements - which may be driven, for example, by the degradation kinetics of the devices.

d) Use the same materials or substances in contact with the same human tissues or body fluids during implantation life (see Meddev 2.7/1 revision 4 PDF document at). Part 6 of International Standard ISO 10993 (2016 version) describes how to evaluate the local tissue effects of absorbable implants including polyester devices - during their full implantation life, i.e. until their complete degradation [7]. Histological analyses of absorbable implants should be performed as a minimum at the following points:

a) Early timeframe; where there is no or minimal degradation,

b) Mid timeframe; when the degradation is taking place and

c) Late timeframe; when the implant is essentially absorbed [7].

The standard even recommends evaluating the device when its fragmentation occurs to allow for the assessment of the tissue response when it is expected to be the most pronounced [7]. Knowing that long-lasting polymer such as PLA could take more than years to completely degrade [8]; in vitro pre-degradation is presented as an option to permit faster but still predictable assessment of long-term tissue reaction, since it does not substantially altering the polymer behavior, in particular its local tissue effects [7,9]. Materials can be then pre-degraded to induce a substantial loss of the polymer mass for example up to $50 \%$ of the implant original weight [7].In vitro incubation at elevated temperatures such as $90^{\circ} \mathrm{C}$, is suitable to prepare pre-degraded PLA-based materials equivalent to implants at late stage after in vivo implantation $[10,11]$. However, in such conditions, the implant may be fragmented, losing its original shape and structure, ultimately presenting as particles. Even if this is the expected fate of absorbable materials in their real life, in the body, the fragmented state of the material raises a challenge for the implantation. Particles tend to clump together and form aggregates leading to their encapsulation with possible fibrosis and necrosis consequences, in subcutaneous pockets in rats $[9,12]$. Then, what may be observed can generate misleading results and false alerts on the safety of implants.

When studying orthopedic implants such as plates and rods, directly implanting a compact mass of predegraded material may be still enough representative of the implant shape and therefore of the tissue response, after in situ degradation [13]. However, this should not be the case with thinner and porous materials such as meshed knitted with about $100 \mu \mathrm{m}$ diameter yarns and pores in the millimeter range [14]. After pre-degradation, such materials should have lost their original shape and come in particles. Currently, for IS010993 part 6 studies, it is still an open challenge to shorten the duration of implantation in a reasonable manner (e.g. less than 1 year), by using pre-degraded materials while generating predictable and clinically relevant data on local tissue effects.

The objective of the present study was to define an appropriate method to obtain a relevant evaluation of tissue response to absorbable implants, i.e. open knitted meshes made of polyester yarns, at their late degradation stage. To prevent their aggregation during implantation, pre-degraded meshes coming as particles were embedded in a collagen film. And this formulation vs free particles were compared in terms of local tissue effects, after subcutaneous implantations, in rabbits, up to 52 weeks.

\section{Materials and Methods}

Absorbable polyester (Poly(L-lactide) or Poly(L-lactide) trimethylene carbonate) knitted textiles (Medtronic - Sofradim Production, France) were sterilized by Ethylene Oxide and, then, pre-degraded aseptically.The results included in this work were obtained from 2 implantation series evaluating predegraded materials, the first one performed with free-particles (FP) and the second with same particles embedded in collagen film (PIC).

\section{"Free-Particles" (FP) Sample Preparation}

Poly(L-lactide) (PLLA) manufactured by NatureWorks (USA) was extruded into $100 \mu \mathrm{m}$ and $150 \mu \mathrm{m}$ diameter yarns by Monosuisse (Romania). Both yarns were used to knit a textile in 
which, after sterilization, samples $(2 \times 1.5 \mathrm{~cm})$ were aseptically cut and in vitro pre-degraded to prepare FP samples. Textile samples were placed in containers (Sterile $50 \mathrm{ml}$ Falcon tubes, Revol France, Ref DU352098) with pre-heated $90^{\circ} \mathrm{C}$ standard phosphate buffer (neutral pH 7.4). Tubes were hermetically closed and incubated at $90^{\circ} \mathrm{C}$ for 120 hours. At the end of pre-degradation, tubes were centrifugated and buffer was removed by aspiration. Containers with pre-degraded samples were placed at $4^{\circ} \mathrm{C}$ until implantation. Control tubes were prepared in parallel to evaluate gravimetric mass retention. For these samples, containers and textile samples were weighed before degradation, washed, dried and weighed after degradation. Weights were verified daily until reaching stability (i.e. $+/-2 \%$ between two consecutive weights). The gravimetric mass retention percentage was calculated using initial and final weight.

\section{“Particles-in-Collagen” (PIC) Samples Preparation}

Poly(L-lactide) trimethylene carbonate (PLLA-TMC) (Medtronic, USA) was extruded into $125 \mu \mathrm{m}$ and $150 \mu \mathrm{m}$ diameter yarns. Both yarns were used to knit a textile in which, after sterilization, samples ( $5 \mathrm{~cm}$ diameter) were aseptically cut and predegraded in vitro to prepare PIC samples. Samples were placed in polytetrafluoroethylene (PTFE) container (Steam sterilized PTFE container 120mL; VWR, ref. 216-0167) with approximately $100 \mathrm{~mL}$ of pre-heated $90^{\circ} \mathrm{C}$ standard phosphate buffer (neutral $\mathrm{pH} 7.4$ ). Containers were hermetically closed and incubated at $90^{\circ} \mathrm{C}$ for 90 hours. At the end of pre-degradation buffer was carefully removed by aspiration and approximately $2 \mathrm{~mL}$ of buffer was kept. Similarly, as for FP samples, control samples were prepared, dried and weighted to evaluate gravimetric mass retention.

A 3\% porcine oxidized collagen (US2001008930 (A1) 2001-0719 - US6391939 (B2) 2002-05-21) was adjusted with sterile water to obtain final concentrations of collagen, Polyethylene Glycol (PEG 4000-VWR Ref 8.17063.5000) and glycerol (EP, USP compliant anhydrous glycerol-VWR Ref 1040931000) of 2.7\%, $0.9 \%$ and $0.54 \%$, respectively, at $\mathrm{pH} 5$ and $38^{\circ} \mathrm{C}$. Three $\mathrm{ml}$ of the prepared collagen solution was added to each container and textile particles were immediately manually homogenized within the solution. Containers were then placed horizontally and remained still on the lab bench, at room temperature, for at least one hour. When the collagen solution was jellified, containers were placed in humidity and temperature regulated climatic chamber for overnight drying $\left(20^{\circ} \mathrm{C}-40 \% \mathrm{HR}\right)$. After drying, samples for implantation $(2 \mathrm{~cm}$ diameter) were cut, packed and sterilized (Ethylene Oxide).

\section{Animal study}

Animal studies were performed in a Contract Research Organization (NAMSA Lyon, Chasse/Rhône, France), in accordance to the European Directive 2010/63/UE on the Protection of Animals used for Scientific Purposes. The study protocols were reviewed and approved by the Contract Research Organization Ethical Committee.
New Zealand White male rabbits (Charles River, France), weighing between $3.0-3.8 \mathrm{~kg}$, were used during the in vivo studies. Rabbits were anesthetized (intramuscular injection of ketamine and xylazine at $10 \mathrm{mg} / \mathrm{kg}$ and $0.1 \mathrm{mg} / \mathrm{kg}$ respectively), placed in a ventral recumbency position. Dorsal areas were clipped and prepared for aseptic surgery. Anesthesia was maintained by inhalation of an $\mathrm{O}_{2}$ and isoflurane mixture (IsoFlo ${ }^{\circledR}$, Axience, 0.5-5\%). Each rabbit received eight $2 \mathrm{~cm}$-long cutaneous incisions (four per side) parallel to the spine. Eight subcutaneous pockets were created by blunt dissection in the subcutaneous tissue and one sample was placed in each subcutaneous pocket. FP samples were placed in subcutaneous pockets using a spatula. Native and PIC samples were introduced in the subcutaneous pockets using hemostatic forceps. When possible, samples were fixed in their central upper side with a non-absorbable stitch (SurgiPro ${ }^{\mathrm{TM}}$ II 5-0, Medtronic USA) to avoid articles folding and facilitate article's location at termination.Once all samples were placed in the subcutaneous pockets, the skin incisions were closed with stainless steel skin staples (Appose ${ }^{\mathrm{TM}}$ ULC Auto Suture ${ }^{\mathrm{TM}}$ - Slim Body Skin Stapler 35W, Medtronic USA) and light pressure bandages were applied over the dorsal area of the rabbits. The staples and bandages were removed 2 weeks following surgery.

At designated time-points (4 weeks, 10 weeks, 26 weeks and 52 weeks) after implantation, rabbits were euthanized using a lethal injection of pentobarbital solution. Final numbers of sites per group and per time-period are summarized in Table 1. The subcutaneous tissues were excised, sampled and flat fixed in $10 \%$ buffered formalin solution. After complete fixation, the tissue explants were dehydrated in alcohol solutions of increasing concentration, cleared in xylene and embedded in paraffin. Sections were prepared using a microtome $\left(\right.$ MICROM $^{\circledR}$, France) and stained by Safranin-Hematoxylin-Eosin and modified Masson's Trichrome.

Table 1: Number of sites analyzed per group and per timeperiod.

\begin{tabular}{|c|c|c|}
\hline \multicolumn{2}{|c|}{ FP study } & PIC study \\
\hline Native - 4W & 10 & 10 \\
\hline Native - 52W & 9 & 10 \\
\hline Particles- 10W & $\mathrm{NA}^{*}$ & 10 \\
\hline Particles- 26W & 11 & 7 \\
\hline Particles- 52W & 9 & 10 \\
\hline
\end{tabular}

Note: *10-week time-period was not evaluated for FP particles.

\section{Analyses}

At necropsy, samples presence and shape were observed. Histological sections were digitized with a Zeiss Zen 2012 SP2 v1.0 slides scan which was used to histomorphometrically measure the product. Mean particle size was determined measuring the diameter of maximum 10 particles amongst the largest ones visible on 
site. Only particles with a cylindrical shape were selected to reflect the particle diameter as much as possible. Mean particle diameter gives indirect indications on material resorption over time, as mean particle size which was used by Bergsma [13].

The implant area was defined as being the surface area of the residual material plus the surface area of the local inflammatory reaction elicited by the implant. It gives indications on the product distribution and on the extent of the material resorption and associated cell reaction over time.Descriptive statistic tools were used to represent the implant area distribution per group. R studio version 3.4.4 software was used to calculate the $1^{\text {st }}$ and $3^{\text {rd }}$ quartiles of each group. The difference between the 1 st and the $3^{\text {rd }}$ quartile (interquartile range) reflects the homogeneity of the values. Using the same software, a one sample t-test, with $\boldsymbol{\alpha}$-risk $=0.05$, was performed to compare the particle diameters of each group to the native yarn diameter $(150 \mu \mathrm{m})$. Similarly, a 2-sample t-test, with $\alpha$-risk $=0.05$, was performed to compare the particle diameters of each group over time.Qualitative histological evaluation of the local tissue effects and material degradation of each implanted site was

Table 2: Implant area and particle size measurements. performed by a qualified pathologist using a microscope Nikon Eclipse 80i.

\section{Result}

\section{Sites Implanted with Native Samples}

At necropsy, no significant macroscopic changes were observed at the sites implanted with native samples. Histomorphometrically, implant areas issued from native groups were homogenous in both series of implantation as shown by the small interquartile ranges obtained (Table 2). Mean implant areas of native sites did not decrease with time. Implants were markedly integrated into the adjacent tissues up to 52 weeks (Fig.1). They were surrounded by a consistent slight to moderate granulomatous tissue over time, mainly showing an interfacial infiltration of macrophages and giant cells (Figures 1a-1b). Well integrated implants (i) and remodeled subcutaneous connective tissue. A normal foreign body reaction $\left(^{*}\right)$ mostly constituted of macrophages and multinucleated foreign body giant cells surrounded the mesh yarns. No significant signs of material degradation detected.

\begin{tabular}{|c|c|c|c|c|c|c|}
\hline \multicolumn{4}{|c|}{ FP study } & \multicolumn{3}{|c|}{ PIC study } \\
\hline \multirow{3}{*}{ Native - 4W } & Implant Area & & \multirow{2}{*}{$\begin{array}{c}\text { Mean Particle Size } \\
(\mu \mathrm{m})\end{array}$} & Implant Area & & Mean Particle Size \\
\hline & $\operatorname{Mean}\left(\mathrm{mm}^{2}\right)$ & Interquartile Range & & Mean $\left(\mathrm{mm}^{2}\right)$ & Interquartile Range & $(\mu \mathrm{m})$ \\
\hline & 3.3 & 0.9 & $150 *$ & 6.7 & 1.4 & $150^{*}$ \\
\hline Native - 52W & 4 & 1 & $150^{*}$ & 6.7 & 1 & $150^{*}$ \\
\hline Particles- 10W & NA & $\mathrm{NA}$ & NA & 14.4 & 6.6 & $83^{* *}+/-24 / 90 N$ \\
\hline Particles- 26W & 8.8 & 7.2 & $151+/-15 / 110 N$ & 4.3 & 1.7 & $52^{* *} \pm 28 / 52 \mathrm{~N}$ \\
\hline Particles- 52W & 3.2 & 6.3 & $140+/-32 / 23 N$ & 2.4 & 1.3 & $0 \times$ \\
\hline
\end{tabular}

Note: *mean diameter of the largest yarn

** significantly different from the native value $(150 \mu \mathrm{m}), \mathrm{p}$ value $<0.005$

$\mathrm{N}$ number of particles analyzed (max 10 particles/site)

×only intracytoplasmic material visible

NA: not applicable as no site

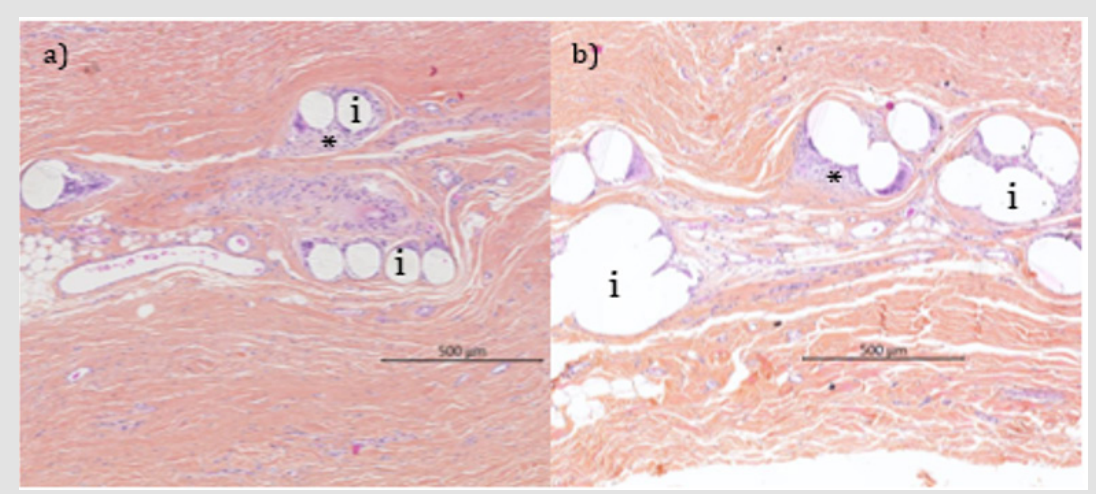

Figure 1: Histological micrographs of native implants respectively used in the FP (a) and PIC (b) studies at 52 weeks (Safranin Hematoxylin Eosin stain).

Note: Well integrated implants (i) and remodeled subcutaneous connective tissue. A normal foreign body reaction (*) mostly constituted of macrophages and multinucleated foreign body giant cells surrounded the mesh yarns. No significant signs of material degradation detected. 


\section{Sites Implanted with FP Samples}

Accelerated pre-degradation of meshes has generated particles with $59 \%$ of the initial sample mass which were then spread as homogeneously as possible in subcutaneous pockets in rabbits.After 26 weeks of implantation, particle heaps were macroscopically visible at all FP sites. At 52 weeks, they were only observed at 4 sites out of 9 . Histomorphometrically, on tissue sections (4-6 $\mu \mathrm{m}$ ), implant areas were not homogenous as shown by the larger interquartile ranges obtained (Table 2). Although the mean implant area decreased from 26 weeks to 52 weeks, the mean particle diameter did not differ significantly from that of the native yarns ( $p$ value 0.76 at 26 weeks, $p$ value 0.16 at 52 weeks) and that

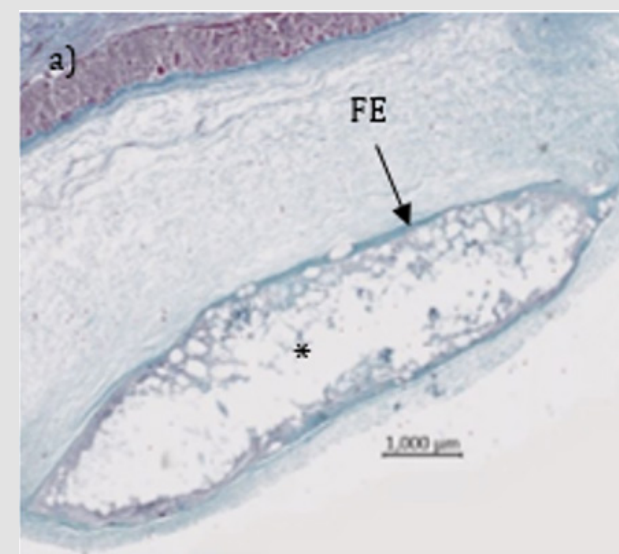

it did not decrease significantly with time between 26 and 52 weeks ( $\mathrm{p}$ value 0.15 ).

At 26 weeks, the large variation observed in implant areas was explained by the presence of dense areas of particles at 4 sites out of 11 as observed by histomorphometry on the analyzed tissue sections. These dense areas were characterized by very limited inter-particle spaces, poor cellular influx (along with signs of necrosis) and development of a dense fibrous capsule (Figure 2a). At sites where particles were not encapsulated (7 out of 11), the implant areas were not consistent among the sites since their thickness varied from $300 \mu \mathrm{m}$ to $800 \mu \mathrm{m}$ as the result of particle stacking (Figure 2b). A non-homogeneous tissue ingrowth along with a moderate interfacial granulomatous reaction was observed.

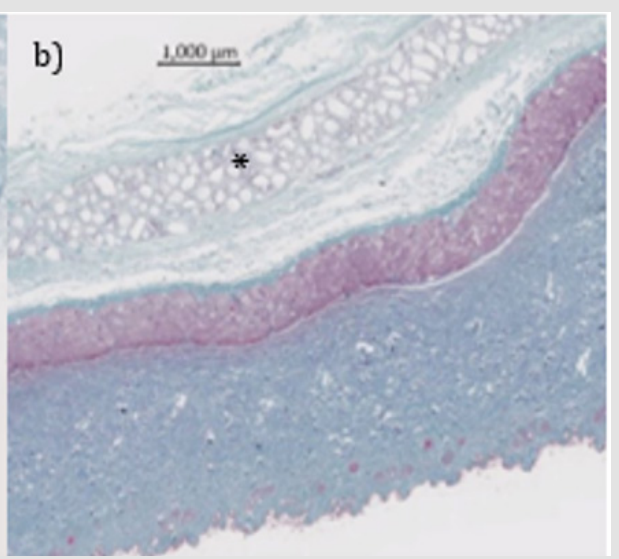

Figure 2: Histological micrographs of FP sites at 26 weeks (Masson trichrome stain).

Note: Variable distribution of the free particles resulting either in dense area of particles $\left(a^{*}\right)$ with evidence of a fibrous encapsulation (FE) and a poor tissue ingrowth, or in less compacted particles $\left(b^{*}\right)$, here integrated despite the thicker layer compared to the native mesh structure of Figure 1 (a).

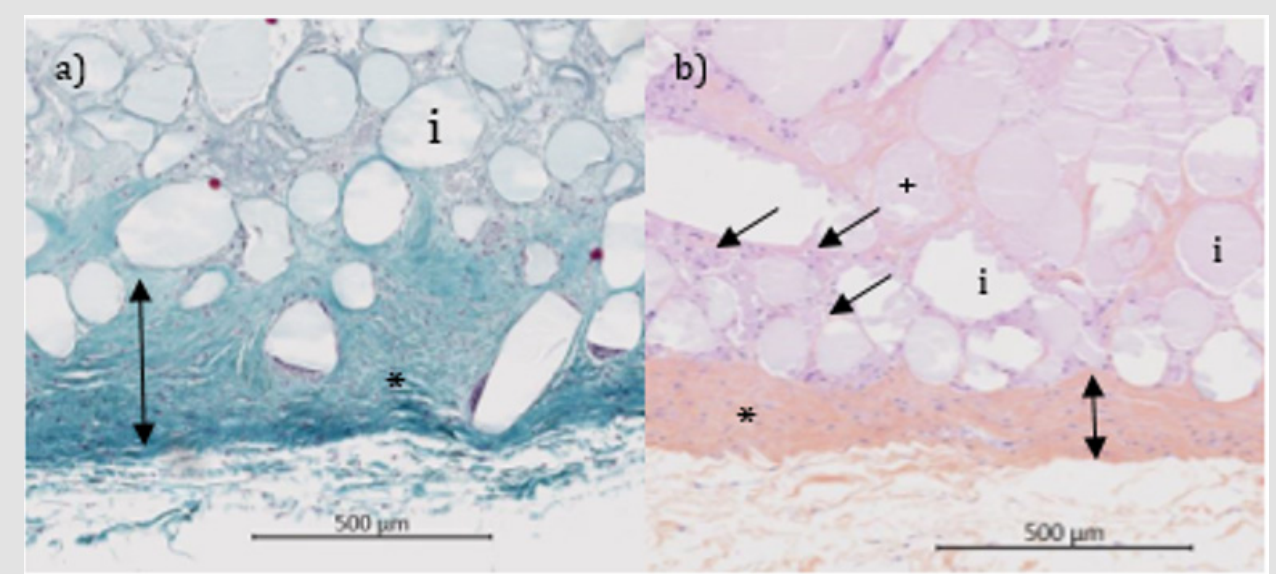

Figure 3: Histological micrographs of FP sites at 26 weeks (a, Masson trichrome stain) and 52 weeks (b, Safranin Hematoxylin Eosin stain).

Note: Fibrous encapsulation of dense area of particles associate with a poor cellular influx at 26 and even a lower one at 52 weeks (double-headed arrow). The particles size was almost unchanged over time while signs of bulk erosion (+) and local acidic toxicity to cells (lower cell viability and increased necrosis (arrow) were more observed at 52 weeks. The fibrous capsule $\left.{ }^{*}\right)$ isolating the implant mass (i) prevented neutralization and elimination of acidic by-products by the cells and body fluid (including buffering effects). 
After 52 weeks, it was hard to sample tissue sections with remaining particles, due to the loss marks of original implantation sites and particle aggregation. Consequently, histology observations of FP sites ( $n=9)$ appeared heterogenous with 1 site showing only a few particles, 2 sites presenting dense areas of stacked particles and 7 sites with no implant material microscopically visible. When densely grouped, the particles still appeared walled-off by a fibrous capsule. The individual particles displayed signs of bulk erosion even though no noticeable dimensional changes were reported (Figure 3). Some of the occasional cells infiltrating the dense areas displayed signs of karyorrhexis (necrotic pattern). Fibrous encapsulation of dense area of particles associate with a poor cellular influx at 26 and even a lower one at 52 weeks (double-headed arrow). The particles size was almost unchanged over time while signs of bulk erosion $(+)$ and local acidic toxicity to cells (lower cell viability and increased necrosis (arrow) were more observed at 52 weeks. The fibrous capsule (*) isolating the implant mass (i) prevented neutralization and elimination of acidic by-products by the cells and body fluid (including buffering effects).

\section{Sites Implanted with PIC Samples}

Accelerated in vitro pre-degradation led to particles with $48 \%$ of the initial sample mass. Then the particles were mixed with collagen to get films with satisfying even distribution of particles.After implantation, materials were visible at 10 weeks, barely visible at 26 weeks and not-visible at 52 weeks.At 10 weeks, microscopically, no residue of collagen disc material was detected. As expected, at 10 weeks, the mean implant area obtained was higher than that of native samples due to the foreign body reaction (Table 2). At 26 and 52 weeks, the interquartile ranges obtained were comparable to those obtained with native samples, meaning that a homogeneous distribution among the sites was obtained (Table 2). A significant decrease of mean particle size was measured compared to the native ones ( $\mathrm{p}$ value $<0.01$ at 26 and 52 weeks) and over time between 10 and 26 weeks ( $p$ value $<0.01$ ). At 26 weeks, PIC sites showed a marked tissue ingrowth and tissue integration. PIC sites were observed as a long band of inflammatory cells admixed with small residual particles (Figure 4a). A moderate granulomatous reaction (mostly macrophages and giant cells) along with marked signs of cell-mediated material degradation was observed. Negligible areas with stacked particles could be detected. The remnants were integrated into the surrounding tissues. And no fibrous encapsulation as well as no signs of necrosis were noted from 26 weeks onwards. At 52 weeks, the tissue ingrowth and integration of a marked grade were associated with a slightly decreased granulomatous reaction (Figure 4b). Detection of extracellular remnants was difficult and most of the sites (6 sites out of 9) showed only intracytoplasmic material (Figure 4c). For that reason, particle size was not recorded anymore. These findings indicated a near complete degradation of the implant at 52 weeks.

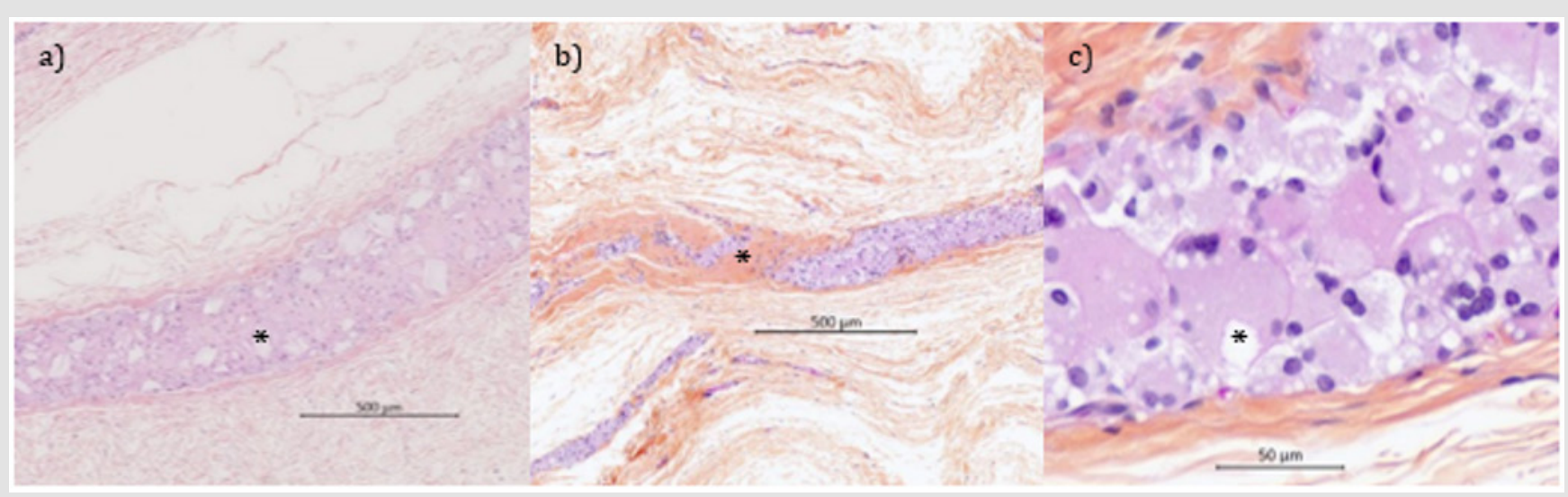

Figure 4: Histological micrographs of PIC sites at 26 weeks (a) and 52 weeks (b and c) (Safranin Hematoxylin Eosin stain). Note: Inflammatory response to small residual particles $\left(a^{*}\right)$ showing on-going signs of degradation. Residual inflammatory response progressively replaced by a dense fibrous $\left(b^{*}\right)$. PIC material completely degraded with only intracytoplasmic accumulation of material left $\left(\mathrm{c}^{*}\right)$ without any signs of cytotoxicity at the late interval.

\section{Discussion}

The implantation lifetime of slowly degrading absorbable polymers cannot be easily investigated in animal studies, unless including pre-degraded polymers as starting materials for implantation. As an example, in a 5-year implantation study in bone, small fragments of PLA plates were still visible 8. After 6 months of implantation, Phasix ${ }^{\mathrm{TM}}$ Mesh (Bard, USA) made of poly-4-hydroxybutyrate (P4HB) is still substantially present in a porcine hernia model and delivering mechanical support of the abdominal wall defect $[15,16]$. Similar behavior was observed with TIGR®Matrix (Novus Scientific, Sweden). This device is a two-component fiber mesh - slow degrading fiber made of Poly(L-lactide) trimethylene carbonate and fast degrading fiber made of Poly(glycolide-co-lactide) trimethylene carbonate. Slow degrading fibers were not completely absorbed with still visible residues after 1-year implantation in a rabbit hernia model [17] and has disappeared after 3 years in a functional model in sheep [18]. Therefore, the use of pre-degraded implants, as suggested in 
standard ISO 10993-6, can be a clinically relevant and predictive approach for the evaluation of local tissue effects throughout of the life of implants.

This approach has been investigated in this study with meshes based on PLLA and Poly(L-lactide) trimethylene carbonate with comparable degradation times (i.e from 2 to 5 years) and mechanisms (i.e. bulk hydrolysis) $[4,17,18]$. What should be then observed with one polymer should also occur most probably with the second one. The series of implantation shown here were selected from all studies done by the authors to support more head to head comparisons, notably in terms of pre-degradation protocols and time points of the preclinical study. After in vitro pre-degradation, polyester meshes lose their structure and come into particles whatever their composition, PLLA and Poly(L-lactide) trimethylene carbonate. When implanted as free particles (FP), aggregates are observed with the formation of dense areas. Such conditions are known to be unfavorable to cell infiltration which may lead to fibrous encapsulation [19]. This situation can worsen with local acid $\mathrm{pH}$ shift due to the formation of water-soluble monomers and oligomers within the fibrous capsule where the flow of extracellular fluids is too low to achieve the local $\mathrm{pH}$ homeostasis [9].

The combination of the fibrous capsule and the local acidity isolate even more the particles from the cellular response, vascular supply and local tissue environment [20]. These unfavorable conditions caused cell lysis or necrosis as observed in our study. This resonates with clinical situations where tissue necrosis and osteolysis were observed a long time after the implantation of dense material such as orthopedic fixation devices (e.g. rods, pins, screws) made of PLLA [21]. By contrast, macroporous (i.e. pores of $1 \mathrm{~mm}$ or more) abdominal hernia meshes are generally well integrated with tissue growing through the pores and with minimal complications in terms of fibrous capsule formation and tissue necrosis [22]. When degraded in situ, for example up to three years after implantation referring to TIGR® Matrix18, no dense packing of the material has been observed spaces between yarns well maintained. So, the results obtained with FP samples, mostly leading to densely packed and encapsulated particles, cannot appropriately predict the foreign body reaction profile. In other words, pre-degradation of meshes may lead to erroneous results. The initial features of the mesh should be preserved, particularly the porosity or interspace between particles to better mimic the degradation course of native meshes and the associated tissue response.

It has been thus proposed here to study an alternative formulation, with the inclusion of homogeneously dispersed pre-degraded mesh particles in an oxidized collagen film (PIC formulation) to prevent stack formation. This film material has been selected for its high biocompatibility and fast degradation time, with full disappearance after one-month implantation [23]. It is already used in Parietex ${ }^{\mathrm{TM}}$ Composite meshes (Medtronic) with good pre-clinical and clinical outcomes [23-25]. The collagen film has been consistently reported to be degraded in about 1-month 13,26]. It is then expectedthat the collagen film will not interfere with the foreign body reaction elicited by PLLA and Poly(L-lactide) trimethylene carbonate, even in their pre-degraded presentation. And that is exactly what was observed in this study from macroscopic and histology analysis. Using a similar approach, Rozema et al. [26] had implanted PLA particles in gelatin capsules or in hydroxypropyl-methyl cellulose gel to observe the final stage of the cellular degradation process. They have seen no difference of cellular response between the two tested formulations up to 16 weeks after implantation. It should be noted that in Rozema's study the implantation vehicles were degraded $48 \mathrm{~h}$ after implantation, which could have been too short to insure a persistent proper particles distribution in vivo.

In our study, using the same in vitro pre-degradation technique, only PIC samples finally permit to closely mimic in situ degradation and to evaluate the complete degradation process of the particles. Enough interspace between particles was maintained to foster tissue ingrowth, neovascularization, cellular response and activity and extracellular fluid circulation. Moreover, a significant decrease of the mean particle size with time was observed at PIC sites. This means that the late stage degradation of materials with their elimination from implantation site was initiated. At FP sites, such observations were not made, most probably due to insufficient flow of fluids in the space of the particles. It should be noted that particle size was determined using the diameter measured on the largest cylindrical-shaped particles. The high standard deviation observed on mean particle size value is directly linked to the limits of histological sections which run through irregular shape particles13. Histological pictures of PIC sites have clearly shown the presence of intracytoplasmic materials, confirming the critical role of phagocytes in polymer absorption [27]. When sufficiently reduced by hydrolysis, the resorption of PLLA particles is accelerated by cellular phagocytosis $[9,28]$. This cell-mediated elimination process can be the cause of late adverse reaction in patients26. That is the reason why the safety profile of absorbable implants should be addressed during all their life implantation time, until their disappearance or the stabilization of the foreign body reaction over months, in relevant preclinical models.

\section{Conclusion}

Collagen embedding of pre-degraded polymer particles before implantation led to i) a more homogenous product distribution and ii) the elucidation the product degradation profile up to the final stage of degradation. Our pre-degraded absorbable meshes have been advantageously included in collagen films, providing appropriate particles distribution, closer to what is observed after implantation of long-term fully absorbable meshes. Finally, it permitted to recapitulate the local inflammatory response from what is known in the literature during the full lifetime of implants, until the end-stage events, cellular phagocytosis and disappearance 
of extracellular materials. And this is perfectly in alignment with the ISO 10993-6 requests [7]. The present study emphasized i) the need of pre-degraded samples to address the safety of long-term absorbable devices in a reasonable time \& ii) the importance of the formulation of pre-degraded materials to mimic as far as possible the "natural" evolution of the shape of materials during their implantation. And the learnings of this study should surely help any clinical development of slowly degrading materials which have to comply with stricter regulatory requirements.

\section{Acknowledgment}

We would like to thank Ludovic Boure, Philippe Gravagna and Robert Vestberg for their critical review of the manuscript and their valuable input. We further thank Delphine Petit and Boris Grange for their support with the experimental work.

\section{Funding}

The study was funded by Medtronic.

\section{Conflict of interest}

Emilie Payet, Cécile Beausseron-Valentin, Thierry Brune, Amandine Radlovic, and Yves Bayon are employees of Medtronic. Studies were designed and performed using sound scientific methods and standardized measures for impartial data collection and comparison.

\section{References}

1. Zhang Z, Ortiz O, Goyal R,Kohn J (2013) Biodegradable Polymers Handbook of Polymer Applications in Medicine and Medical Devices 303-336.

2. Pawar RP, Tekale SU, Shisodia SU, Totre JT,Domb AJ(2014) Biomedical Applications of Poly(Lactic) Acid. Recent Pat Regen Med 4: 40-51.

3. Athanasiou KA, Niederauer GG, Agrawal CM (1996) Sterilization, toxicity, biocompatibility and clinical applications of polylactic acid/polyglycolic acid polymers. Biomaterials 17: 93-102.

4. Ramot Y, Zada MH, Domb AJ, Nyska A(2016)Biocompatibility and safety of PLA and its copolymers. Adv Drug Deliv Rev 107: 153-162.

5. Weir NA, Buchanan FJ, Orr JF, Farrar DF, Boyd A(2004) Processing, annealing and sterilisation of poly-L-lactide. Biomaterials 25(18): 3939 3949.

6. Carrasco F, Pagès P, Gámez-Pérez J, Santana 00, Maspoch ML(2010) Processing of poly (lactic acid): Characterization of chemical structure thermal stability and mechanical properties. Polymer Degrad. Stab 95(2): 116-125.

7. (2016) Biological evaluation of medical devices. Part 6: Tests for local effects after implantation.

8. Suuronen R, Pobjonen T, Hietanen J and Lindqvist C(1998) A 5-year in vitro and in vivo study of the biodegradation of polylactides plates. J Oral Maxillofac Surg 56: 604-614.

9. De Jong WH, Bergsma JE, Robinson JE,Bos RRM(2005) Tissue response to partially in vitro predegraded implants. Biomaterials 26: 1781-1791.

10. Lyu S, Schley J, Loy B, Lind D, Hobot C, et al. (2007) Kinetics and Time -Temperature Equivalence of polymer degradation. Biomacromol 8 2301-2310.
11. Bergsma JE, Rozema FR, Bos RRM, Boering G, Joziasse CAP, et al. (1995) In vitro predegradation at elevated temperatures of poly(lactide). J Mater Sci Mater Med 5: 575-581.

12. Rozema R, Bergsma JE, Bos RRM, Van Roendaal AWM, De Jong WH, et al. (1995) Biocompatibility and degradation mechanisms of predegraded and non-predegraded poly(lactide) implants: an animal study. J Mater Sci Mater Med 6: 715-724.

13. Bergsma JE, Rozema FR, Bos RRM, Boering G, De Bruijn WC, et al. (1995) In vivo degradation and biocompatibility study of in vitro pre-degraded as polymerized polylactide particles. Biomaterials 16: 267-274.

14. Rastegarpour A, Cheung M, Vardhan M, Ibrahim MM, Butler CE, et al. (2016) Surgical mesh for ventral incisional hernia repairs: Understanding mesh design. Plast Surg 24: 41-50.

15. Scott JR, Deeken CR, Martindale RG, Rosen MJ (2016) Evaluation of a fully absorbable poly-4-hydroxybutyrate/absorbable barrier composite mesh in a porcine model of ventral hernia repair. Surg Endosc 30(9): 3691-3701.

16. Martin DP, Badhwar A, Shah DV, Rizk S, Eldridge SN, et al. (2013) Characterization of poly-4-hydroxybutyrate mesh for hernia repair applications. J Surg Res 184(2): 766-773.

17. Peeters E, Van Barneveld KW, Schreinemacher MH, De Hertogh G, Ozog Y et al. (2013) One-year outcome of biological and synthetic bioabsorbable meshes for augmentation of large abdominal wall defects in a rabbit model. J Surg Res 180: 274-283.

18. Hjort H, Mathisen T, Alves A, Clermont G, Boutrand JP (2012) Three-year results from a preclinical implantation study of a long-term resorbable surgical mesh with time-dependent mechanical characteristics. Hernia 16: 191-197.

19. Bryers JD, Giachelli CM,Ratner BD (2012) Engineering biomaterials to integrate and heal: the biocompatibility paradigm shifts. Biotech Bioeng 109(8): 1898-1911

20. Anderson JM (2001) Biological responses to materials. Annu Rev Mat Res 31: 81-110.

21. Böstman OM, Pihlajamäki HK (2000) Adverse Tissue Reactions to Bioabsorbable Fixation Devices. Clin. Orthop. Relat. Res 371: 216-227.

22. Amid PK (1997) Classification of biomaterials and their related complications in abdominal wall hernia surgery. Hernia 1: 15-21.

23. Mutter D, Jamali FR, Moody DL, Rodeheaver GT, Thérin M,et al. (2000) The concept of protected mesh to minimize adhesion formation in intraperitoneal abdominal wall reinforcement. Preclinical evaluation of a new composite mesh. Hernia 4: S3-S9.

24. Balique JG, Benchetrit S, Bouillot JL, Flament JB, Gouillat C, et al. (2005) Intraperitoneal treatment of incisional and umbilical hernias using an innovative composite mesh: four-year results of a prospective multicenter clinical trial. Hernia 9: 68-74.

25. Bellón JM, García Carranza A, Jurado F, García Honduvilla N, Carrera San Martin A, et al. (2001) Peritoneal regeneration after implant of a composite prosthesis in the abdominal wall. World J Surg 25: 147-152.

26. Rozema FR, Bergsma JE, Bos RRM, Boering G, Nijenhuis AJ, et al. (1994) Late degradation simulation of poly(l-Lactide). J Mater. Sci Mater Med 5: 575-581.

27. Anderson JM, Rodriguez A, Chang DT(2008)Foreign body reaction to biomaterials. Semin Immunol 20: 86-100.

28. Ozdl D, Wimpenny I, Aydin HM,Yang Y (2017) Biocompatibility of biodegradable medical polymers. In: Science and Principles of Biodegradable and Bioresorbable Medical Polymers: 379-414. 
ISSN: 2574-1241

DOI: 10.26717/BJSTR.2019.24.003987

Yves Bayon. Biomed J Sci \& Tech Res

(c) (P) This work is licensed under Creative

Submission Link: https://biomedres.us/submit-manuscript.php

BIOMEDICAL
RESEARCHES $\quad \begin{aligned} & \text { Assets of Publishing with us } \\ & \text { - Global archiving of articles }\end{aligned}$

